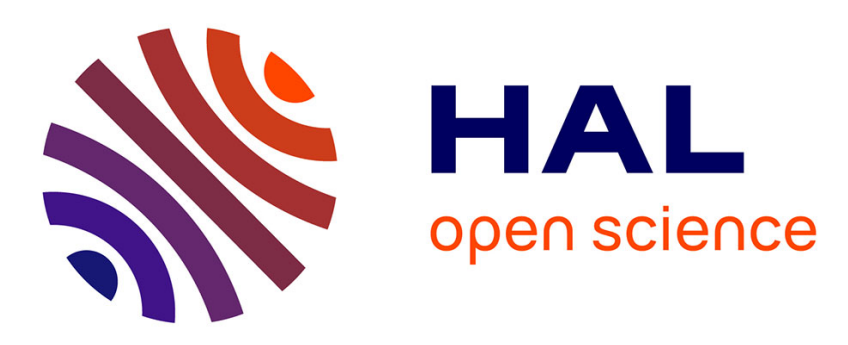

\title{
Experimental characterization of nonlinear static and dynamic behaviors of circular capacitive microplates with initial deflection
}

Aymen Jallouli, Najib Kacem, Gilles Bourbon, Patrice Le Moal, Joseph Lardies

\section{To cite this version:}

Aymen Jallouli, Najib Kacem, Gilles Bourbon, Patrice Le Moal, Joseph Lardies. Experimental characterization of nonlinear static and dynamic behaviors of circular capacitive microplates with initial deflection. Nonlinear Dynamics, 2021, 103 (3), pp.2329 - 2343. hal-03414230

\author{
HAL Id: hal-03414230 \\ https://hal.science/hal-03414230
}

Submitted on 4 Nov 2021

HAL is a multi-disciplinary open access archive for the deposit and dissemination of scientific research documents, whether they are published or not. The documents may come from teaching and research institutions in France or abroad, or from public or private research centers.
L'archive ouverte pluridisciplinaire HAL, est destinée au dépôt et à la diffusion de documents scientifiques de niveau recherche, publiés ou non, émanant des établissements d'enseignement et de recherche français ou étrangers, des laboratoires publics ou privés. 


\title{
Experimental characterization of nonlinear static and dynamic behaviors of circular capacitive microplates with initial deflection
}

\author{
Aymen Jallouli • Najib Kacem • Gilles \\ Bourbon • Patrice Le Moal • Joseph \\ Lardies
}

Received: date / Accepted: date

\begin{abstract}
In this paper, the effects of initial deflection on the static and dynamic behaviors of circular capacitive transducers are experimentally investigated. The obtained results are in good agreement with numerical simulations. It is shown that the initial deflection has a major impact on the static response of the resonator by shifting the pull-in voltage, and on its dynamic response by increasing the resonance frequency and modifying the bifurcation topology from softening to hardening behavior. Moreover, the dynamic behavior of the microplate may display nonlinear periodic and quasiperiodic responses due to geometric and electrostatic nonlinearities.
\end{abstract}

Keywords electrostatic resonator $\cdot$ geometric imperfections $\cdot$ experimental characterization · nonlinear dynamics · bifurcation topology

PACS 05.45.-a $\cdot 07.07 . \mathrm{Mp} \cdot 46.40 . \mathrm{Ff}$

\footnotetext{
Aymen Jallouli, Najib Kacem, Gilles Bourbon, Patrice Le Moal and Joseph Lardies Univ. Bourgogne Franche-Comté, FEMTO-ST Institute, UMR 6174, CNRS/UFC/ENSMM/UTBM, Department of Applied Mechanics, F-25000, Besançon, France

E-mail: najib.kacem@univ-fcomte.fr
} 


\section{Introduction}

Nano and microelectromechanical systems (NEMS/MEMS) are commonly made from microbeams and microplates [35]. These type of resonators are used in several applications such as logic devices [14], microswitches [19,12], microphone [24, 13,48], mass and gas sensors [40,11,45,34,33] and biological and microfluidic applications [6,3]. However, these micro devices are subject to some imperfections due to a residual stress gradient caused by the microfabrication process $[49,10]$. Despite the fact that the geometric imperfections can be very small compared to the size of the micro-system; it has a major effect on its dynamic and static responses. Initial imperfections have been the focus of researchers in the last few years $[46,1,3]$.

The majorities of the researches were focusing on the dynamic and static responses of beams with initial deflection. For cantilevers, the residual stress changes the shape of the beam to a curved shape [31]. This initial deflection has a major effect on the pull-in threshold depending on the bending direction, which can cause the failure of the microstructure when the two electrodes comes into contact $[49,50]$. Several studies have been also conducted on microbeams with clamped-clamped boundary condition thanks to its rich dynamic and static behavior (snap-through, bistablity, pull-in ).

Younis et al $[51,49,50]$ and Ouakad [32] presented the effect of the initial deflection on the nonlinear dynamic and static behavior of a clamped-clamped beam such as softening and hardening behavior, dynamic snap through and dynamic pull-in. From experimental point of view, curved polysilicon microbeam can be used as bandpass filters and low-powered switches. The effects of axial force resulting by the residual stress or temperature variation have been investigated by Wang et al [47]. The governing equation of motion has been discretized using the Galerkin method, the obtained equations have been solved using the method of multiple scale to investigate the dynamic response of the beam in the presence of 
three-to-one internal resonance by obtaining the frequency response, force response time history phase plane portrait, Poincare section and Fourier spectrum.

The nonlinear behavior of a micro-system has been also studied for the case of a microplate with initial deflection. Saghir et al. [37-39] developed the von Kármán plate equation that introduces the electrostatic and geometric nonlinearities. The Galerkin method has been used to discretize the obtained partial differential equations. The numerical model, supported with experimental tests, have been used to study the static and dynamic behaviors of the microplate. They showed the effect of the DC voltage on the static deflection and the natural frequency. Under a harmonic $\mathrm{AC}$ voltage, the microplate can have a hardening behavior, due to the effect of the midplane stretching nonlinearity, and a softening behavior due to the effect of the electrostatic nonlinearity. The effects of initial deflection on the natural frequencies have been studied numerically. The increase of the initial deflection leads to an increase of the first natural frequency until it reaches a maximum before it starts decreasing. However the higher modes showed a steady increase. Other studies $[8,9]$ investigated the effects of initial deflection of a rectangular plate under different boundaries conditions by presenting an approximate solution for the free flexural vibration.

For the case of circular microplate, Medina et al [25-27] developed a reduced order model (ROM) of an initially curved circular plate actuated with electrostatic force. Their studies showed that due to the electrostatic nonlinearity this kind of MEMS structure can have a snap-through. To transform the ROM to a more compact one, they used Berger's approximation for which a single mode can be used to predict the bistable behavior for the case of low thickness to gap ratios microplate.

Zhang [52] derived the equation of motion of deflected circular plate using the principle of virtual work by taking into account the in-plane tension. A new approximated static analytical solution is derived and it showed a much simpler 
and more direct framework to study the plate-membrane transition behavior compared to other analytical approaches.

The nonlinear behavior of circular plates was also investigated at the macroscale by Touzé and Thomas [44,43]. The equations of motion of the circular plate with free edge boundary condition were derived by taking into account the plate imperfection. The analytical model was validated with respect to experimental results obtained by measuring the deflections of the antinode. The nonlinear frequency responses of the vibration amplitude and the phase were determined for different configurations. By estimating the input parameter of the system, the developed model is capable to predict the nonlinear phenomena of the plate.

In our previous works $[16,17]$, a computational model was developed that investigates the nonlinear static and dynamic behaviors of circular (Capacitive Micromachined Ultrasonic Transducers) with initial imperfection by transforming the von Kármán equation into a set of ordinary differential equations using the differential quadratic method. The model showed a good performance in predicting the nonlinear behavior of the circular microplate and its bifurcation topology.

Most of the previous experimental studies in MEMS actuators have been focusing on beams or rectangular shape microplate with initial imperfection. In this paper, an experimental nonlinear characterization of an electrostatic resonator with circular shape and initial deflection is presented. The main objective in this context is to highlight the influence of low initial deflection on static and dynamic behaviors of circular microplates. First the fabrication technique of the microplates was optimized in order to reduce the residual stress and minimize geometric imperfections. For the second part, different experimental techniques are used during the characterization of the microplate. For the static characterization, an optical interferometer type Mirau was used to measure the topography of the microplate for different DC voltages and to present the effect of the initial deflection on the static behavior and the pull-in voltage of the resonator. For the dynamic characterization, a Laser Doppler Vibrometer was used to measure 
the velocity of the microplate surface while changing the frequency. From these experimental results, we presented the effects of the initial deflection on the linear eigenfrequencies of the resonator and its nonlinear frequency response. Also, The dynamic behavior of the microplate is investigated by looking to its time history and Fourier spectrum.

\section{Fabrication of the resonator and identification of its design parameters}

\subsection{Fabrication process}

The resonator is fabricated using anodic bonding technique, where the microplate and the gap are defined on different wafers and bounded at low temperature and by applying a voltage difference [5]. The Figure 1 presents the process flow used in this work to fabricate the microsystem. The fabrication process was executed in MIMENTO clean room according to the following steps: we start with a wafer of glass Borofloat 33 and a Silicon On Insulator wafer (SOI) with a $2.3 \mu \mathrm{m}$ silicon device layer representing the membrane thickness.

The first step in the process is creating the gap in the glass wafer. A thin layer of chromium and gold is deposited on the surface of the wafer, Figure 1a. The gold layer is used as a hard mask for glass etching. Next, the cavity shape is defined by photolithography patterning of a previously coated resin layer and etching of the gold / chromium layers. The wafer is then etched with a buffered hydrofluoric acid solution (BHF) to create the cavity, Figure 1b. After creating the cavity, the hard mask is removed by putting the wafer into a gold and chromium etch solution.

A thin layer of gold was deposed at the cavity to create the bottom electrode of the resonator using the lift off technique, Figure 1c. The bottom electrode thickness has to be controlled because the corresponding deposited metal reduces the electrostatic gap. 
The two wafers are placed in the bonding machine at a very low pressure of $10^{-2} \mathrm{~Pa}$ and heated to $350{ }^{\circ} \mathrm{C}$ since the two wafers have an equal CTE $350{ }^{\circ} \mathrm{C}$ which is very convenient for the bonding. Once the temperature of the two wafers is stabilized, a force of $1000 \mathrm{~N}$ is applied followed with four steps of voltage (300 $\mathrm{V}, 500 \mathrm{~V}, 700 \mathrm{~V}$ and $900 \mathrm{~V}$ ) for $10 \mathrm{~min}$ each to limit the peak current [5]. The two wafers are now bonded and a controlled cooling started, Figure 1d. The controlled cooling is very essential to release the stress created during the bonding.

In the next step, the handle layer is removed using $\mathrm{KOH}$ solution. The oxide layer protect the silicon device layer from etching. Thereafter, the buried oxide layer is removed with BHF solution, Figure 1e. Next, a thin aluminum layer is deposited on the remaining silicon device layer. Another key concept to reduce the residual stress is to perform a thin and slow deposition rate of the aluminum electrode (the thickness is $150 \mathrm{~nm}$ and the deposition rate $49 \mathrm{~nm} / \mathrm{min}$ ). The aluminum layer has two important roles: first it is used as a hard mask for the etching of the device layer defining membranes and second it is used as a top electrode on the membranes. The aluminum layer is etched after the photolithography patterning of a previously coated resin layer. Finally, the silicon device layer is etched by Reactive Ion Etching using the aluminum hard mask to define membranes and access to the bottom electrode, Figure $1 f$.

\subsection{Design parameter identification of the resonator}

The microplate, as it is presented in figure 2 is mainly composed of three parts:

- the membrane, with radius $R$ and a thickness $h$, which is the movable part that generates the acoustic waves.

- the bottom electrode, with radius $R_{e l e}$, representing the fixed electrode which delimits the action area of the electrostatic forces (excluding edge effects).

- the gap, with height $d_{g}$, which represents the distance between the top and the bottom electrode. 


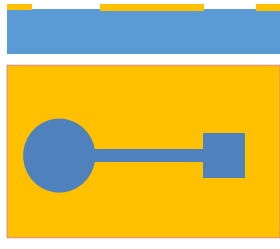

(a)

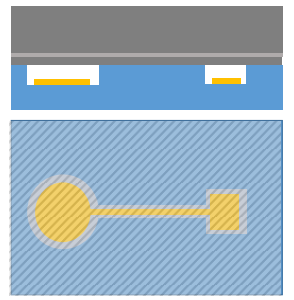

(d)

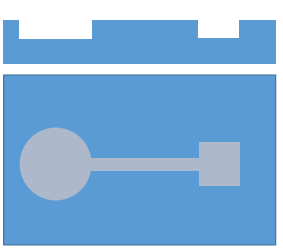

(b)

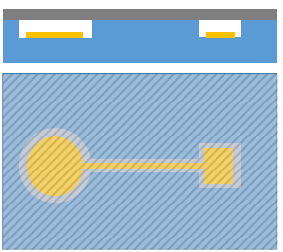

(e)

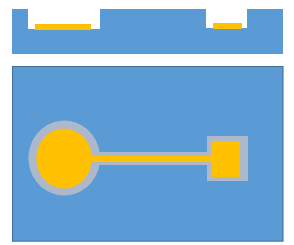

(c)

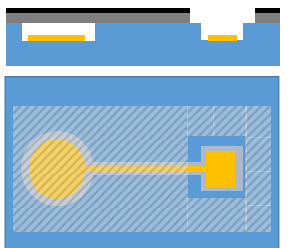

(f)

Gold Au

Glass (Borfloat)

Silicon Si

Aluminium Al

Fig. 1: The process flow of the anodic bonding method. (a) Deposition and patterning of the gold / chromium layer (b) Etching of the cavity in the glass wafer, (d) deposition of the bottom electrode, (d) anodic bonding of the glass wafer with the SOI wafer, (e) etching of the silicon substrate and the buried oxide of the SOI, (f) deposition of the top electrode and dry etching of the membrane.

The plate is a bilayer Silicon / Aluminum but the stiffness (given by the thickness and the Young modulus) of the aluminum layer can be neglected compared to that of the silicon. As the silicon is a brittle material at room temperature [15], the material of the plate can thus be considered elastic and homogeneous. Moreover, the circular structure by its axisymmetric nature allows to suppose the material of the plate as isotropic with an average Young modulus in the plane (100) around $149 \mathrm{GPa}$ between two extremal values $130 \mathrm{GPa}$ and $169 \mathrm{GPa}$. The microplate is subjected to an axisymmetric initial deflection $w_{0}(r)$ that can be approximated as follows:

$$
w_{0}(r)=w_{\max } \frac{1+\cos \left(\frac{\pi r}{R}\right)}{2},
$$

where $w_{\max }$ represents the initial deflection of the membrane at its center. 


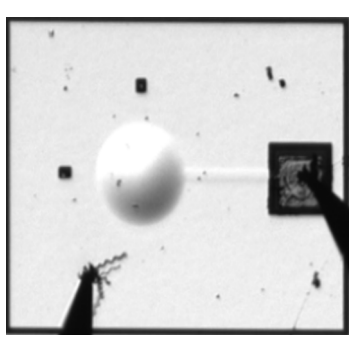

(a)

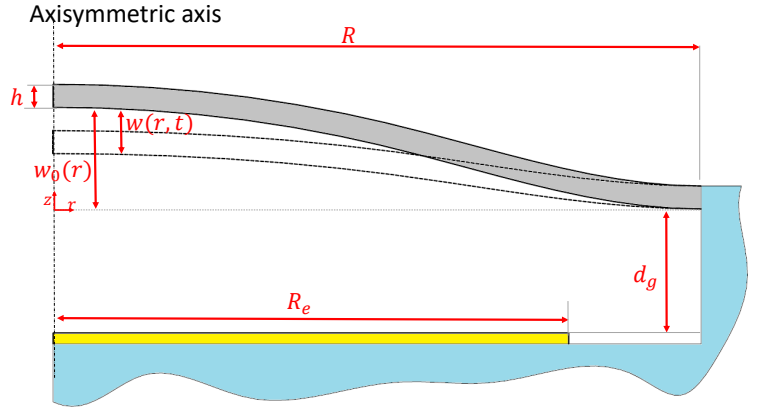

(b)

Fig. 2: (a) A photo of the micro-device after fabrication. (b) A cross section of a circular electrostatic thin microplate.

It is important before starting the characterization to measure the dimensions of the micro-device like the diameter, the gap distance, the electrode thickness and the membrane thickness. To obtain these values, we used an optical microscope to measure the lateral dimensions (like the radius of the cavity and the bottom electrode) and a contact profilometer for vertical dimensions (like the thickness of the membrane, the depth of the cavity and the thickness of the bottom electrode). The design parameters of the micro-system are displayed in Table 1 with the errors reported for the measured dimensions.

Table 1: Physical parameters of the micro-system

\begin{tabular}{ccc}
\hline Symbol & Quantity & Dimension \\
\hline$E$ & Youngs modulus & $149[\mathrm{GPa}]$ \\
$\rho$ & Density & $2330\left[\mathrm{~kg} / \mathrm{m}^{3}\right]$ \\
$\nu$ & Poisson's ratio & 0.27 \\
$R$ & Radius of the microplate & $115 \pm 2[\mu \mathrm{m}]$ \\
$R_{e l e}$ & Radius of the bottom electrode & $80 \pm 2[\mu \mathrm{m}]$ \\
$h$ & Thickness of the microplate & $2.3 \pm 0.1[\mu \mathrm{m}]$ \\
$d_{g}$ & Gap distance & $0.6 \pm 0.1[\mu \mathrm{m}]$ \\
\hline
\end{tabular}




\section{Mathematical Model}

We consider a circular microplate excited with an electric voltage $V(t)=V_{d c}+V_{a c} \cos (\omega t)$ where $V_{d c}$ is the DC bias voltage and $V_{a c}$ is the amplitude of the harmonic voltage.

The partial differential equations that describe the dynamic response of the circular microplate, with an initial deflection $w_{0}(r)$, are derived from the von Kármán plate theory [17].

$$
\begin{gathered}
\frac{\rho h^{2}}{D} \ddot{w}+\frac{h^{2}}{D} c \dot{w}+\nabla^{4} w=\frac{N_{0}}{D}\left(\frac{1}{r}\left(w_{, r}+w_{0, r}\right)+\left(w_{, r r}+w_{0, r r}\right)\right)+\frac{\epsilon_{0} V^{2}(t)}{2 D\left(d_{g}-w+w_{0}\right)^{2}} \\
+\frac{12}{h^{2}}\left[\frac{1}{r} u_{, r}\left(w_{, r}+w_{0, r}\right)+u_{, r r}\left(w_{, r}+w_{0, r}\right)+u_{, r}\left(w_{, r r}+w_{0, r r}\right)+\frac{1}{2 r}\left(w_{, r}\right)^{2}\left(w_{, r}+w_{0, r}\right)\right. \\
+w_{, r}\left(w_{, r}+w_{0, r}\right)\left(\frac{1}{r} w_{0, r}+w_{0, r r}\right)+\frac{1}{2}\left(w_{, r}\right)^{2}\left(w_{, r r}+w_{0, r r}\right)+w_{, r} w_{0, r}\left(w_{, r r}+w_{0, r r}\right) \\
\left.+w_{, r r}\left(w_{, r}+w_{0, r}\right)^{2}+\frac{\nu}{r}\left(u_{, r}\left(w_{, r}+w_{0, r}\right)+u\left(w_{, r r}+w_{0, r r}\right)\right)\right] \\
u, \quad 2) \\
u_{, r r}+\frac{1}{r} u, r-\frac{u}{r^{2}}=-\frac{1-\nu}{2 r}\left(w_{, r}\right)^{2}-w_{, r} w_{, r r}
\end{gathered}
$$

where $u(r, t)$ and $w(r, t)$ represent the in-plane and out of plane displacement of the membrane in the $r, \theta, z$ coordinate system. $\nabla^{4}=\left(\frac{\partial^{2}}{\partial r^{2}}+\frac{1}{r} \frac{\partial}{\partial r}\right)^{2}$ the bi-harmonic operator, $D=\frac{E h^{3}}{12\left(1-\nu^{2}\right)}$ the plate flexural rigidity, $c$ the damping coefficient, $(*)_{, r} ;(*)_{, r r}$ first and second derivative with respect to $r$ and $(\dot{*})(\ddot{*})$ first and second derivative with respect to $t$.

For an axisymmetric problem and at the center of the microplate, the out of plane displacement $w$ has always a slope equal to zero and the radial displacement $u=0$. Considering the peripheral clamping of the circular microplate, the boundary conditions are finally [52]: 


$$
\begin{array}{lll}
\text { At } r=0: & w_{, r}=0 & u=0 \\
\text { At } r=R: & w_{, r}=w=0 & u=0 \\
\frac{E h}{1-\nu^{2}}\left(\frac{d u}{d r}+\frac{1}{2}\left(\frac{d w}{d r}\right)^{2}+\frac{\nu}{r} u\right) & =0,
\end{array}
$$

\section{Static response}

\subsection{Static characterization setup}

For static characterization, the wafer is placed underneath the interferometer, Mirau type, to measure the topography of the microplate, as shown in Figure 3. The micro-system is forced by a DC voltage using a DC generator equipped with two probes. This technique can provide accurate $3 \mathrm{D}$ and $2 \mathrm{D}$ scans of the surface of the microplate for different DC voltages. As an advantage of this characterization method, the microplate surface cannot be damaged since the measurements are based on a non-contact scanning using an interferometer. Also, the static displacement of the membrane can be measured while applying a voltage which is not possible with the use of the profilometer.

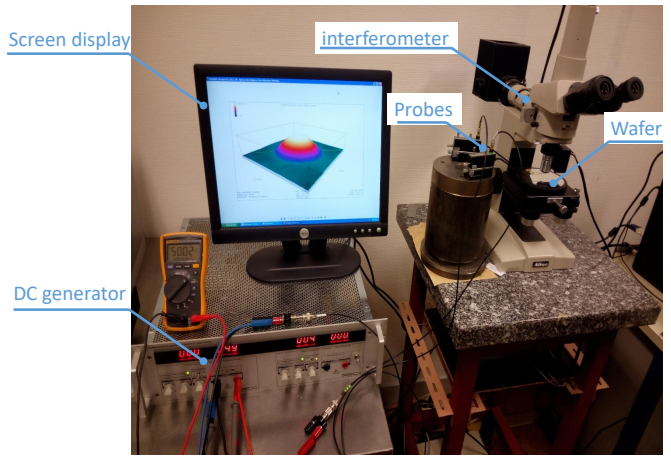

(a)

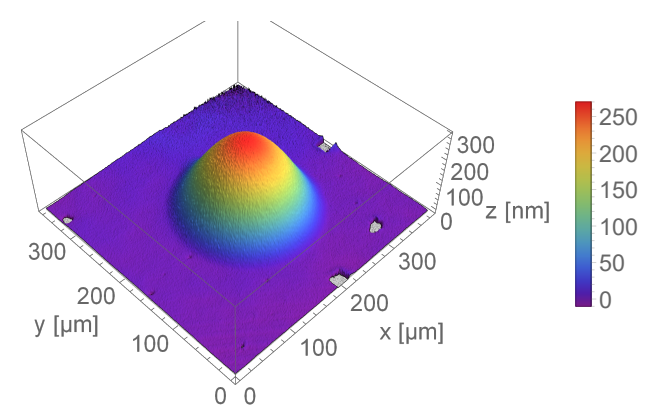

(b)

Fig. 3: (a) Experimental setup for the static response of the resonator. (b) Topography of the membrane surface 
4.2 Experimental results

Figure $4 \mathrm{a}$ and $4 \mathrm{~b}$ present a cross section of the upper surface of the membrane for different DC voltages for the case of flat and initially deflected microplate, respectively. Figure 4a shows clearly that the plate has a flat surface for $V_{d c}=0 \mathrm{~V}$ . By increasing the DC voltage, the microplate starts to bend downward until it reaches its maximum at $V_{d c}=35 \mathrm{~V}$. At this stage, the increase of the electrostatic force can cause the collapse and thus the destruction of the micro-device by shortcircuit, because no insulation layer can prevent the electrical contact between the microplate and the bottom electrode.

The characterized micro-system in Figure $4 \mathrm{~b}$ is initially deflected. In fact, the microplate displays a concave shape initial deflection with a maximum $w_{\max }=270$ $\mathrm{nm}$ for $V_{d c}=0 \mathrm{~V}$. When the electrostatic force increases, the microplate starts to change its form to almost flat for $V_{d c}=49 \mathrm{~V}$, and convex beyond this actuation level.

The static responses of the two micro-system, flat and deflected, are depicted in Figure 4c by presenting the total displacement at the microplate center for different DC voltages. The experimental results are compared to the numerical model presented in [17], which is based on Differential Quadratic Method (DQM). The solid and dashed lines, in Figure 4c, represent the stable and the unstable numerical solutions and the stars $(*)$ marked in this graph correspond to the pull-in points, for which the solution changes from stable to unstable. The experimental static response shows a good agreement with the numerical results obtained from the reduced order model [17]. The maximum error between the numerical and experimental results is less than $5 \%$.

As the DC voltage increases, the total displacement of the microplate increases until it reaches the pull-in point, where the slope of the curve tends to infinity. It can noticed from Figure 4c that an upward initial deflection increases the pull-in voltage. This can be explained by the increase of the gap distance between the two plates. A small initial imperfection $\left(w_{\max }=270 \mathrm{~nm}\right)$ can shift the pull-in voltage 
from $V_{d c}=35 \mathrm{~V}$ to $V_{d c}=53.5 \mathrm{~V}$. Thus, It is important to take into account the effect of initial deflection in the design and fabrication of electrostatic resonators. By analyzing the static response, there is no snap-through behavior since the curve has only one stable branch. This is due to the fact that the initial deflection is small compared to the thickness of the microplate [2,30,23].

\section{Linear and nonlinear dynamic analysis}

\subsection{Dynamic characterization}

The frequency response of the resonator is determined using a vibrometer OFV-534 (Polytec) equipped with a decoder with different precisions (can go up to $1 \mathrm{pm}$ for the displacement and $10 \mathrm{~m} / \mathrm{s}$ for the velocity) and wide frequency range (up to $24 \mathrm{MHz}$ ), shown in Figure 5. The VD02 velocity decoder transforms the optical signal to an electrical one, which will be displayed on the oscilloscope. In order to exhibit nonlinearities (i.e. higher vibration amplitudes), the fluid interactions with the micro-system like ambient air loading and losses including squeeze film effects [18] was eliminated by placing the wafer inside a vacuum chamber, as shown in Figure 5. The electric connections are ensured by two probes placed inside the vacuum chamber. The latter has a transparent window (Plexiglas) enabling the penetration of the laser beam without any distortion. The characterization setup is equipped with two generators in order to excite the resonator with $\mathrm{DC}$ and $\mathrm{AC}$ voltages combined via a coupling circuit.

The construction of the nonlinear frequency response is performed by exciting the microplate with a DC voltage combined with a periodic chirp signal with an amplitude $V_{a c}$. The periodic chirp function is a sinusoidal function that changes its frequency through time defined as follows:

$$
V(t)=V_{d c}+V_{a c} \sin \left(2 \pi f_{0}(t) t\right), \quad \text { where } \quad f_{0}(t)=f_{s}+\frac{f_{e}-f_{s}}{T_{p c}} t
$$




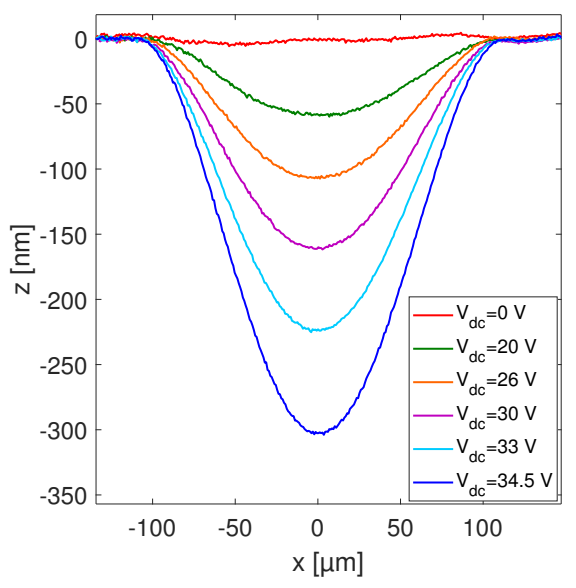

(a)

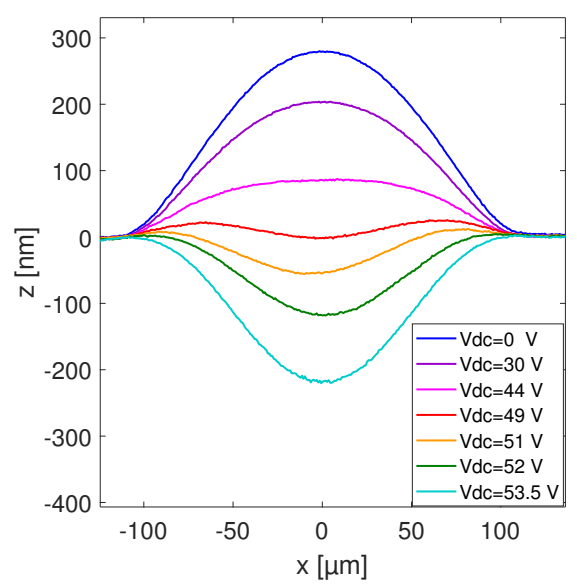

(b)

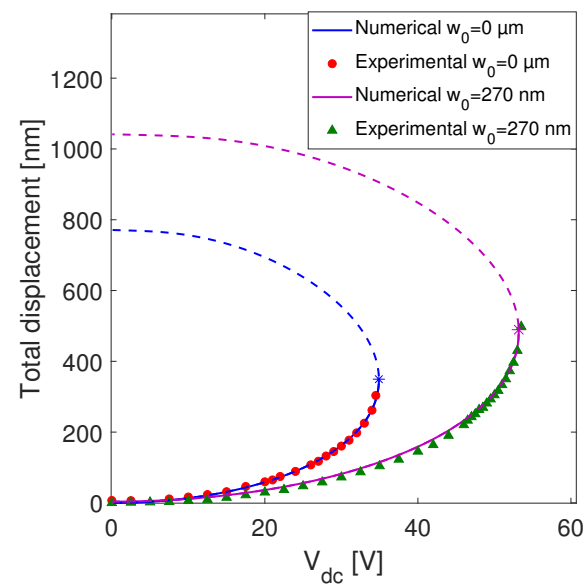

(c)

Fig. 4: (a) and (b) Evolution of the static profile for a clamped circular microplate electrostatically excited for the case of flat and deflected microplate $\left(W_{\max }=270\right.$ $\mathrm{nm}$ ), respectively. (c) Comparison of the static displacement between the analytical model described in [17] and the experimental results. The solid line presents the stable solution of the system and the dashed line presents the unstable solution.

where $f_{0}(t)$ represents the frequency of the sinusoidal function. $f_{s}$ and $f_{e}$ are the starting and final frequencies. $T_{p c}$ is the time taken to sweep from $f_{s}$ to $f_{e}$. The frequency response is then constructed from the time response of the micro-system by plotting the envelope curve of the signal. Figure 6 presents the time response of a softening frequency response of a flat microplate with $V_{d c}=25 \mathrm{~V}$ and $V_{a c}=0.5$ 


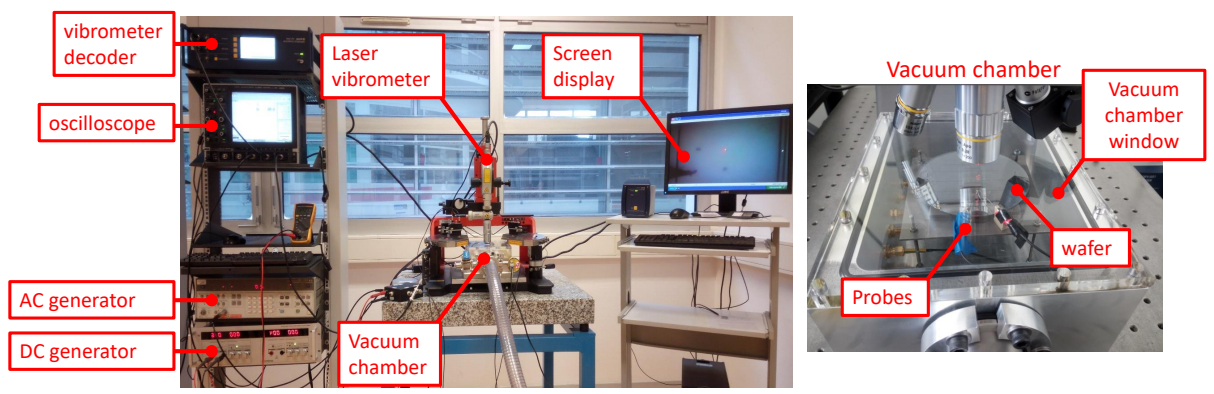

Fig. 5: OFV-534 laser Doppler vibrometer used for nonlinear frequency responses

$\mathrm{V}$ (Figure $6 \mathrm{a}$ and $6 \mathrm{~b}$ represent a forward and backward sweep, respectively). The choice of $T_{p c}$ should be high enough to have a stable time response. This technique is an efficient method to construct stable branches in the bistable domain. The frequency response can be obtained by superposing the two envelope curves of each time response, as it is presented in Figure 6c. In this case the resonator has a softening type behavior because the curve is bent to the left.

\subsection{Linear eigenfrequency analysis}

The initial deflection does not only affect the static response of the microplate, but it also affects its resonance frequencies. In order to determine the variation of the resonance frequencies with respect to the applied DC voltage, a laser vibrometer is used equipped with vacuum chamber, presented in Figure 5. The two circular microplates that has been tested have the same design parameters presented in Table 1, however, one has a flat surface and the other one has an initial deflection $w_{\max }=270 \mathrm{~nm}$.

The nondimensional eigenfrequencies, as it is described in [17], depend mainly on the axial force $N_{0}$, the DC voltage $V_{d c}$ and the initial deflection $w_{0}$. The axial force is chosen by matching the numerical natural frequencies of the microplate, for a low $V_{d c}$, with the experimental ones. For parameters identification, the initial stress and the plate thickness of the microplate are fitted to match the experimental and numerical results (without exciding the error bar presented in table 1) [36,4]. 


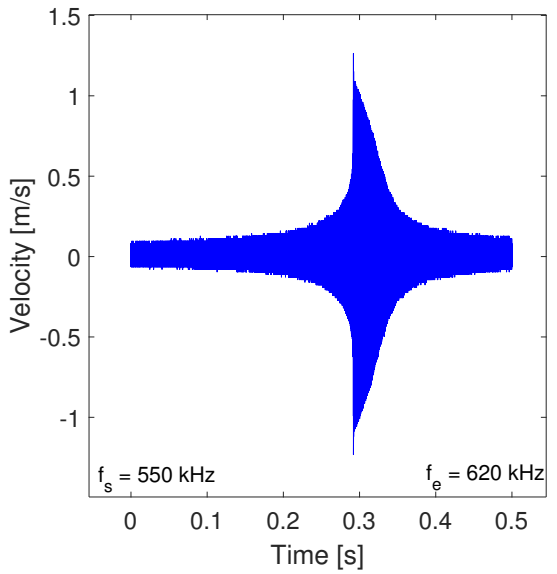

(a)

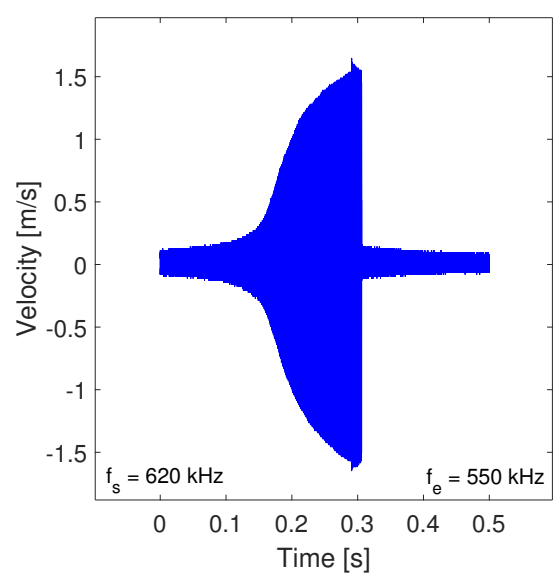

(b)

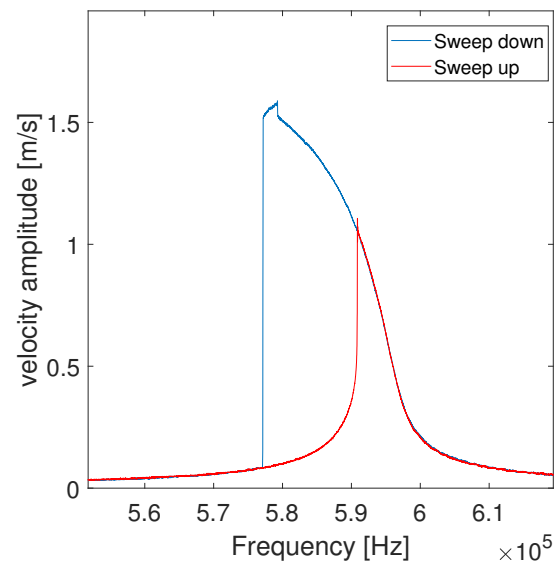

(c)

Fig. 6: Time response of the microplate in the case of (a) forward sweep (from $550 \mathrm{kHz}$ to $620 \mathrm{kHz}$ ) and (b) backward sweep (from $620 \mathrm{kHz}$ to $550 \mathrm{kHz}$ ) (c) the envelop curves of the time response, where $V_{d c}=20 \mathrm{~V}, V_{a c}=0.25 \mathrm{~V}$ and $T_{p c}=0.5$ s.

Therefore, the following error function to minimize can be defined as:

$$
e_{r}=\sum_{i=1}^{2} \operatorname{det}\left(\boldsymbol{K}-\left(2 \pi T f_{e x p, i}\right)^{2} \boldsymbol{M}\right) \text {, }
$$

where $f_{\exp , i}$ is the $i^{\text {th }}$ experimental resonance frequency. In our case, two experimental 
resonance frequencies are used. $\boldsymbol{M}$ and $\boldsymbol{K}$ are the linear mass and stiffness matrix, respectively. $T$ is the nondimensional parameter defined in [17]. The principal axis method [7] is used to determine the plate thickness $h$ and the initial stress $N_{0}$ that minimize the error function $e_{r}$.

The experimental resonance frequencies, displayed in Figure 7, are obtained by exciting the microplate with a small $\mathrm{AC}$ voltage, in order to have a linear response, and different DC voltages. For the numerical results, a mathematical model, developed in [17], was used with the physical parameters presented in Table 1. The nonlinear partial differential equations (2) and (3) were spatially discretized using the Differential Quadrature Method (DQM) to obtain a set of nonlinear differential equations. The nonlinear dynamic behavior of the microplate is studied by discretizing the time variable using the Finite Difference Method (FDM). As it can noticed from Figure 7, the natural resonance frequencies at low $V_{d c}$ of the two resonators are slightly different, this is due to the difference in the residual stress. By increasing the DC voltage, the resonance frequencies decrease due to the increase of the negative electrostatic stiffness which is proportional to $V_{d c}^{2}$. At the pull in voltage, the first resonance frequency drops to zero since the electrostatic stiffness is equal to the mechanical one. Clearly, the numerical model has a good agreement with the experimental data. Figure 7 shows also the influence of the initial deflection on the first resonance frequency. At a constant DC voltage, the initial deflection shifts the resonance frequency of the microplate to higher values. This is due to the increase of the gap distance which reduces the electrostatic force. That means an important DC voltage is needed to bend the microplate.

An other major effect to focus on is the effect of the DC voltage on the dissipated energy of the resonator. Figure 8 shows the measured the quality factor $Q$ of the linear frequency response at different DC voltages using the half-power bandwidth method $\left(Q=\frac{f_{0}}{\Delta f}\right)$. The microplate is actuated with small AC voltage $V_{a c}=0.07 \mathrm{~V}$ in order to have a linear frequency response for different DC voltage. 


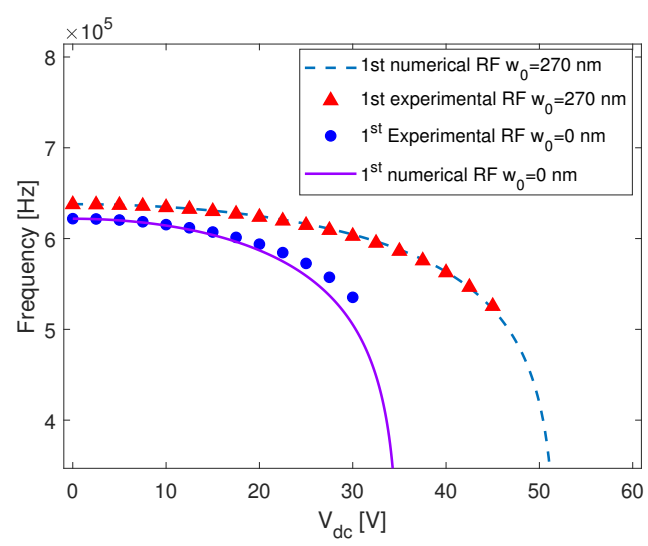

Fig. 7: Comparison between the experimental and analytical results of a flat and deflected microplate of the first resonance frequency

For low DC voltage the quality factor for the two resonators is important since they are in vacuum chamber to avoid air losses. By increasing the DC voltage, the quality factor decreases strongly even at low ratios of DC to pull-in voltages. Losses sources are possibly numerous and discussed in many papers [28] : viscous loss, acoustic radiation, support loss, thermoelastic loss, volume and surface loss,

$$
\frac{1}{Q_{\text {tot }}}=\frac{1}{Q_{\text {viscous }}}+\frac{1}{Q_{\text {support }}}+\frac{1}{Q_{\text {thermoelastic }}}+\frac{1}{Q_{\text {surface }}}+\frac{1}{Q_{\text {ohmic }}}+\ldots
$$

Some of them as gap-closing effect [42] and ohmic losses [41] depends on the DC voltage. However, only the ohmic losses from the electrons moving on and off the resonator due to capacitive coupling to a nearby gate can explain the observed important influence of the DC voltage on the evolution of the quality factor, in particular according to an inverse quadratic variation :

$$
Q_{\text {ohmic }}^{-1}=\frac{1}{\pi \omega} \frac{R_{\Omega}\left(C^{\prime} V_{d c}\right)^{2}}{m_{e f f}}
$$

where $\omega$ is the angular frequency of vibration, $R_{\Omega}$ is the output resistor $C^{\prime}$ is the gradient of the capacitance and $m_{e f f}$ is the effective mass of the resonator. As a 


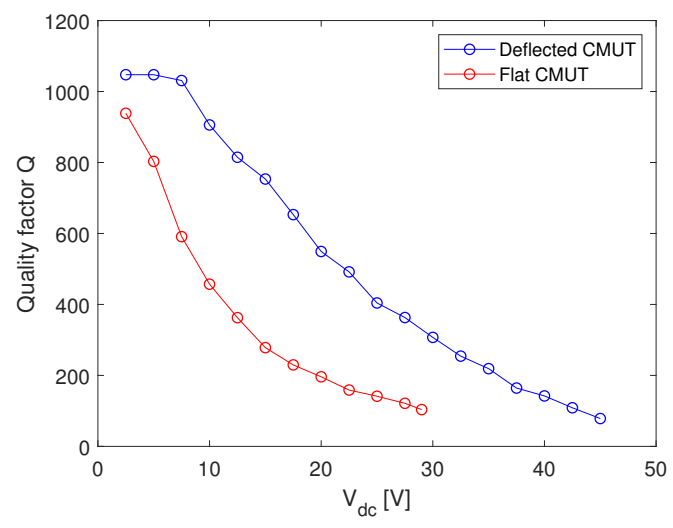

Fig. 8: The experimental quality factor $\mathrm{Q}$ with respect to $V_{d c}$ for the case of a flat and deflected microplate $\left(W_{\max }=270 \mathrm{~nm}\right)$ where $V_{a c}=0.07 \mathrm{~V}$.

conclusion, the more we approach the pull-in voltage of the resonator the more the quality factor decreases.

5.3 Nonlinear frequency analysis

In this section, the nonlinear frequency of a circular microplate is investigated near the primary resonance. In the case of an initially deflected circular microplate, the resonator has two different behavior according to the bias voltage level. For the first case in Table 2, the membrane is excited with a small DC voltage and so the geometric nonlinearities are higher than the electrostatic ones, as shown in Figure 9a. Thus, the frequency response bends to the right displaying a hardening behavior involved by geometric nonlinearities. In the second case reported in Table 2, the micro-system biased by a higher DC voltage shows a softening behavior in Figure 9b. Indeed, the frequency response bends to the left illustrating the predominance of electrostatic nonlinearities over geometric ones [35]. It can be noticed that experimental and numerical results are in a good agreement. The maximum error between the two results is at the bifurcating points. For example the error at the hardening response $9 \mathrm{a}$ is equal $7 \%$ however for the softening case is equal to $12 \%$. This is because at high displacement field induced by nonlinear 
effects other loss sources may occur and the quality factor is more affected than expected. Hardening and softening behaviors are accurately described according to qualitative and quantitative considerations.

Table 2: Excitation parameters used for numerical tests

\begin{tabular}{lccc} 
& $V_{d c}$ & $V_{a c}$ & $Q$ \\
\hline Fist case & $15 \mathrm{~V}$ & $0.25 \mathrm{~V}$ & 700 \\
Second case & $25 \mathrm{~V}$ & $0.25 \mathrm{~V}$ & 500 \\
\hline
\end{tabular}

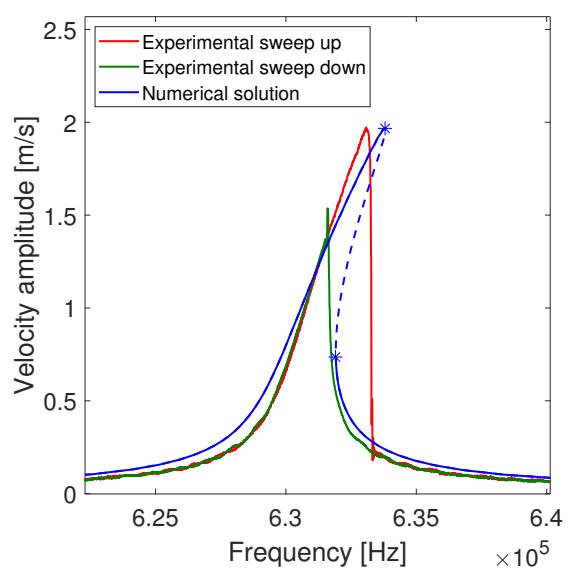

(a)

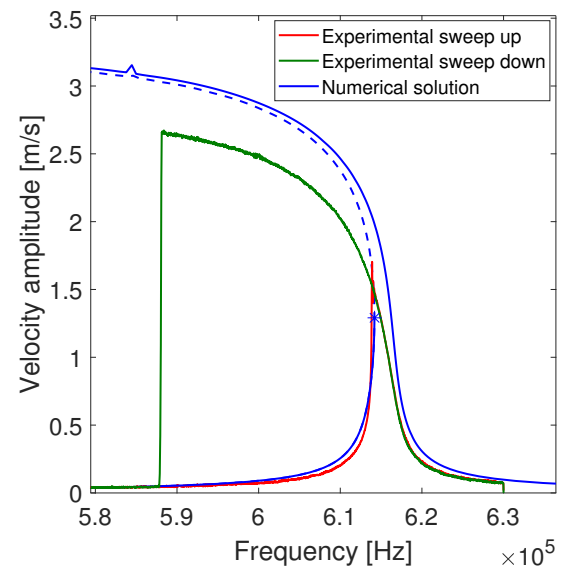

(b)

Fig. 9: Comparison between the experimental and the numerical frequency response of the maximum velocity at the center of the microplate with initial deflection $w_{0}=270 \mathrm{~nm}$ for: (a) small DC voltage, first case in Table 2. (b) for higher DC voltage, second case in Table 2. The solid line presents the stable solution of the system and the dashed line presents the unstable solution.

The nonlinear frequency resonance of the resonator is investigated with respect to DC and AC voltages. In Figure 10a, the microplate was actuated with $V_{a c}=0.25$ $\mathrm{V}$ and different DC voltages. For a low DC voltage $\left(V_{d c}=2.5 \mathrm{~V}\right)$, the microplate vibrates with a low amplitude and the system has a linear behavior. By progressively increasing the DC voltage, the vibration amplitude increases, the resonance peak moves to lower frequency values and the curve bends to the right. As long as 
the bias voltage stays below a threshold of 20V, Figure 10a displays a hardening behavior involved by geometric nonlinearities. Remarkably, at a critical $V_{d c}$ about $20 \mathrm{~V}$, the resonator frequency response changes form hardening to softening due to the amplification of the electrostatic nonlinear terms.

Unlike the effect of $V_{d c}$ on the resonator frequency response, as shown in Figure $10 \mathrm{~b}$ and Figure 10c, the variation of the $\mathrm{AC}$ voltage has a significant impact on the excitation amplitude and a negligible one on the negative electrostatic stiffness. Consequently, the increase in the $V_{a c}$ leads to an increase in the vibration amplitude and the bistability domain without any remarkable frequency shift. This phenomenon is shown in Figure $10 \mathrm{~b}$ for $V_{d c}=5 \mathrm{~V}$ where the frequency response exhibits a hardening behavior. For this case, the geometric nonlinearities are dominating the electrostatic nonlinearities which explains the hardening behavior of the microplate. However, when the electrostatic nonlinearities dominate the geometric nonlinearities, the microplate exhibits a softening behavior as it is shown in Figure 10c.

In Figure 11, we compare the experimental frequency response of a flat and a deflected microplate excited with the same $\mathrm{DC}$ and $\mathrm{AC}$ voltages $\left(V_{d c}=15 \mathrm{~V}\right.$ and $\left.V_{a c}=0.25 \mathrm{~V}\right)$. With a flat surface, the microplate has a softening behavior. Nevertheless, when the microplate has an initial deflection, the behavior of the micro-system changes from softening to hardening. The initial deflection increases the gap distance between the two electrodes which decreases the electrostatic force. In this case, the sign of the overall cubic nonlinearities is positive. Therefore, the frequency response curve bends to the right and exhibits a hardening behavior. Consequently, the bifurcation topology of the resonator frequency response can be tuned with respect to the DC voltage and the initial deflection. Doing so, the performances of the device can be adjusted in terms of generated acoustic power and frequency bandwidth. 


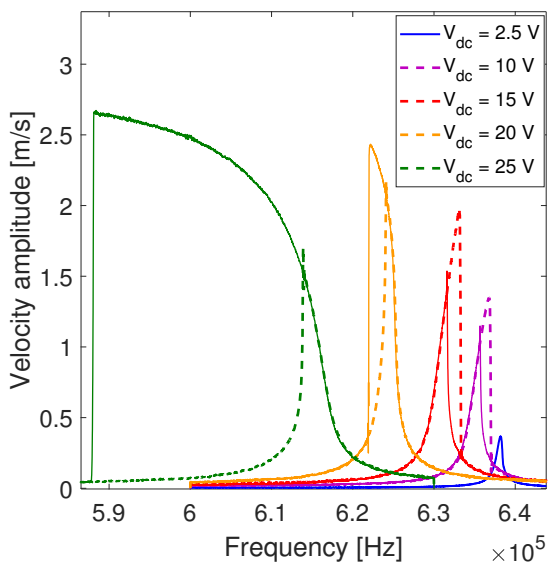

(a)

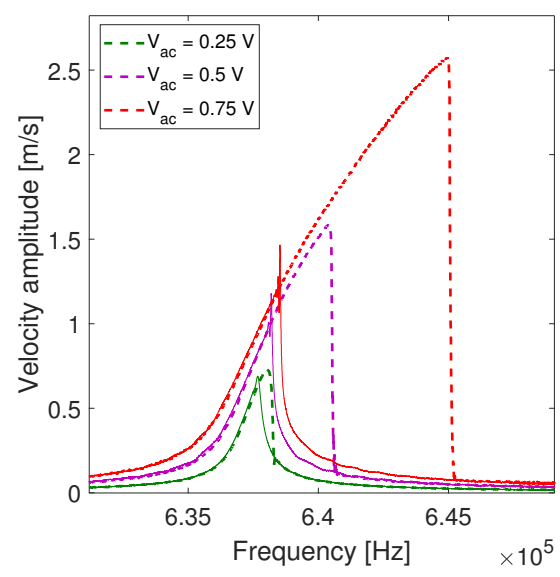

(b)

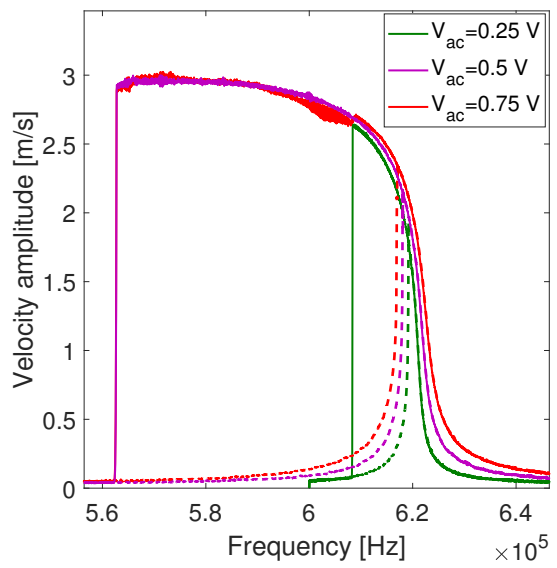

(c)

Fig. 10: Experimental investigation of the effect of the (a) DC voltage $\left(V_{a c}=0.25 \mathrm{~V}\right)$ and $(\mathrm{b}) \mathrm{AC}$ voltage $\left(V_{d c}=5 \mathrm{~V}\right)$ and $(\mathrm{c}) \mathrm{AC}$ voltage $\left(V_{d c}=22.5 \mathrm{~V}\right)$ on the frequency response. The solid and dashed lines represent the vibration amplitude of the microplate by performing a sweep down and a sweep up, respectively.

\subsection{Time history analysis}

Another way to characterize the dynamic behavior of a nonlinear resonator is by analyzing the time history of its response [29]. In this section, we are interested to the upper stable branch of the nonlinear frequency response of the microplate, 


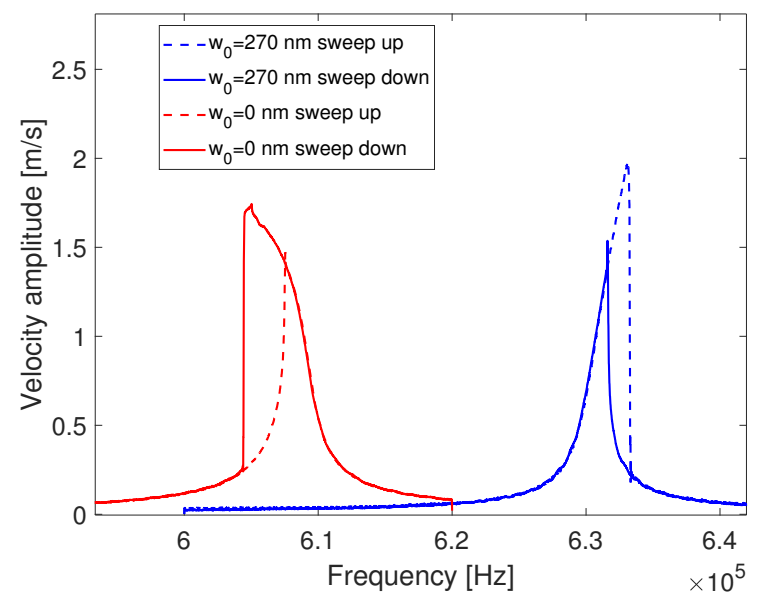

Fig. 11: Experimental frequency response of flat circular microplate and deflected circular microplate with an initial deflection $w_{0}=270 \mathrm{~nm}$, excited with the same voltage $V_{d c}=15 \mathrm{~V}$ and $V_{a c}=0.25 \mathrm{~V}$

Figure 12. the micro-system is actuated with a $V_{d c}=27.5 \mathrm{~V}$ and $V_{a c}=1 \mathrm{~V}$. In this case, the microplate exhibits a softening behavior since the electrostatic force exceeded the critical excitation amplitude [20-22]. The time history of the microplate is determined for different frequencies $\left(f_{a}=605 \mathrm{kHz}, f_{b}=598 \mathrm{kHz}\right.$, $f_{c}=544 \mathrm{kHz}$ and $f_{d}=506 \mathrm{kHz}$ ) by doing a sweep down from $650 \mathrm{kHz}$ to the desired frequency $\left(f_{a}, f_{b}, f_{c}\right.$ and $\left.f_{d}\right)$. Once the right frequency is reached, the time response of the resonator is saved and processed by applying the Fast Fourier Transform (FFT), as shown in Figure 12 (a-d).

In Figure 12a, the excitation frequency is set to be $f_{a}=605 \mathrm{kHz}$, where the frequency response have one stable solution. As it can noticed from the Fourier spectrum, the response contains components of the first and super-harmonics. Decreasing the excitation frequency to $f_{b}=598 \mathrm{kHz}$, which corresponds to a stable response in the bistable region, the Fourier spectrum contains other harmonic peaks around the first and the superharmonics compared to to the frequency $f_{a}$ which explains the quasi-periodic response of the time history response. As shown in Figure 12(c-d), by decreasing more the frequency, the time response becomes 
periodic and the vibration amplitude increases while the signal becomes more distorted when approaching the bifurcation point. The appearance of superharmonics in the time history of the microplate indicates the presence of quadratic and cubic terms originating from electrostatic and geometric nonlinearities.

\section{Conclusion}

The experimental characterization of a circular capacitive transducers and the understanding of their nonlinear behavior were the main focus of this paper.

The micro-system has been characterized with different methods: for the static behavior, an optical interferometer was used to visualize the topography of the upper surface of the microplate for different DC voltages. A comparison between flat and initially deflected resonator was performed showing a good agreement with the theoretical results. Even though this deflection is small, it leads to an important increase of the pull-in voltage due to the increase of the gap distance between the two electrodes.

The dynamic response of the microplate was investigated using Laser Doppler Vibrometer (LDV) by measuring the velocity of the vibrating plate. The DC voltage does not only have an effect on the total displacement of the microplate but also changes its linear eigenfrequencies due to the negative electrostatic stiffness. Also, the increase of the DC voltages leads to an increase of the dissipated energy due to the ohmic losses. The nonlinear dynamic response of the micro-system was also investigated for different DC and AC voltages. The frequency response of the resonator can have both hardening and softening effects depending on the electrostatic force: for low DC voltage the microplate has a hardening type behavior induced by the geometric nonlinearity. The increase in the DC voltage increases the electrostatic nonlinearities and the frequency response changes from hardening to softening. It was shown that a small deflection of the membrane can change the frequency responses from softening to hardening due to the increase of the gap distance between the two electrodes. Moreover, by analyzing the time 

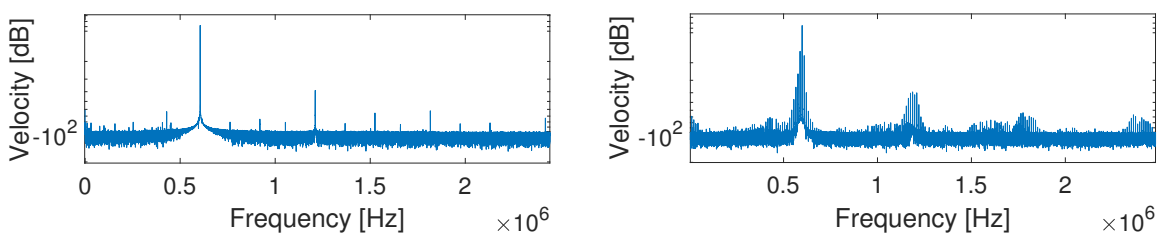

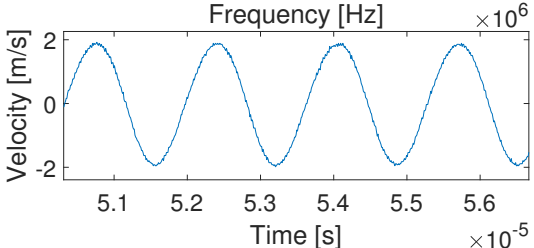

(a)

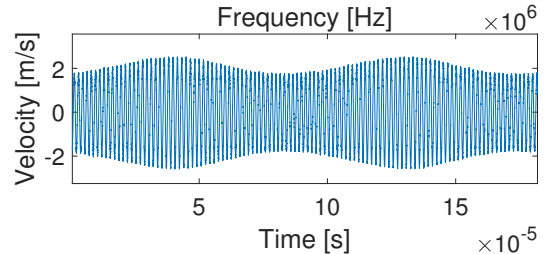

(b)

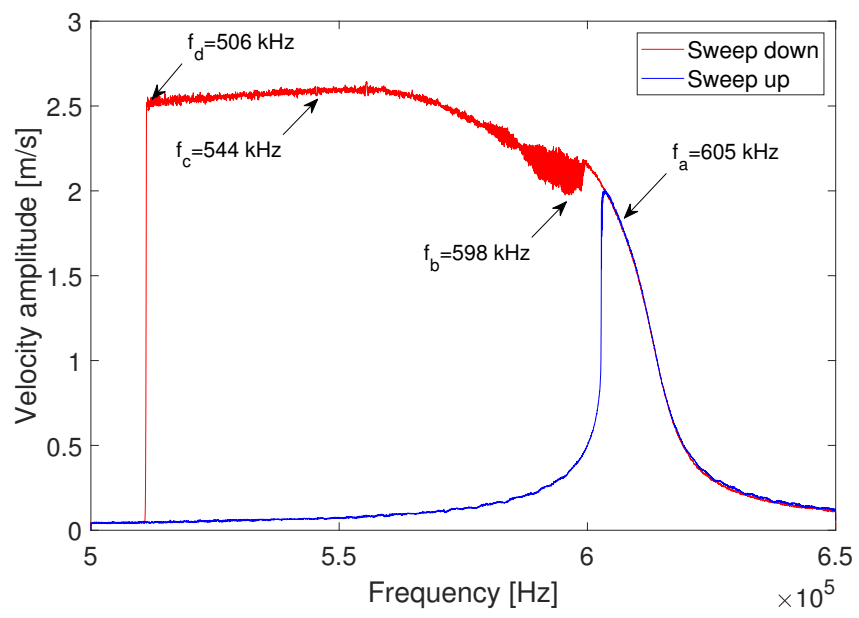

(c)
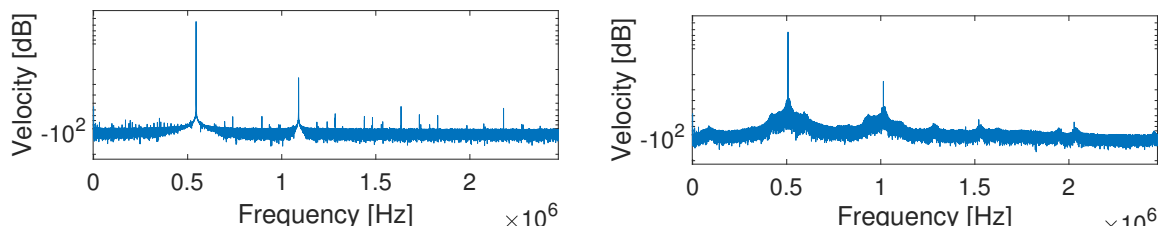

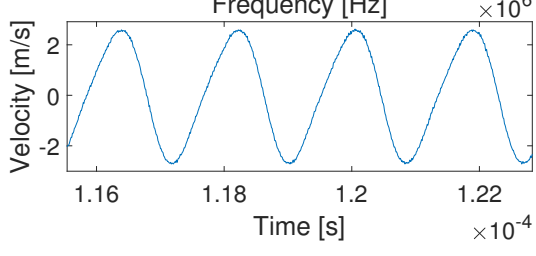

(d)

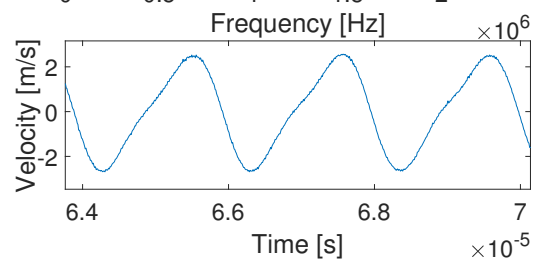

(e)

Fig. 12: (e) Nonlinear frequency responce of the microplate for a $V_{d c}=27.5 \mathrm{~V}$ and $V_{a c}=1 \mathrm{~V}$ (a-d) time response and its FFT for the frequencies $f_{a}=605 \mathrm{kHz}$, $f_{b}=598 \mathrm{kHz}, f_{c}=544 \mathrm{kHz}$ and $f_{d}=506 \mathrm{kHz}$ 
history of the nonlinear frequency response, the resonator showed some interactions between its fundamental harmonic and superharmonics due to quadratic and cubic nonlinear terms coming from geometric and electrostatic nonlinearities.

Even though in this work, circular microplates have been tested in vacuum, future work will include the modeling and the experimental analysis of such devices in air and water for practical applications while taking into account the squeeze film effects.

\section{Acknowledgments}

This project was supported by the Bourgogne-Franche-Comté region and the EUR EIPHI program (ANR 17-EURE-0002).

\section{Declarations}

Funding: This project was funded by the Bourgogne-Franche-Comté region.

Conflicts of interest/Competing interests: The authors declare that they have no conflict of interest.

Availability of data and material: Raw data were generated using the experimental equipments of FEMTO-ST institute. Derived data supporting the findings of this study are available from the first author Aymen Jallouli on request.

Code availability: The code has been implemented using the commercial software Matlab.

\section{References}

1. Alcheikh, N., Hajjaj, A., Younis, M.I.: Highly sensitive and wide-range resonant pressure sensor based on the veering phenomenon. Sensors and Actuators A: Physical 300, 111652 (2019)

2. Alkharabsheh, S.A., Younis, M.I.: Statics and dynamics of mems arches under axial forces. Journal of Vibration and Acoustics 135(2), 021007 (2013) 
3. Alneamy, A.M., Khater, M.E., Abdel-Aziz, A.K., Heppler, G.R., Abdel-Rahman, E.M.: Electrostatic arch micro-tweezers. International Journal of Non-Linear Mechanics 118, $103298(2020)$

4. Bataineh, A.M., Younis, M.I.: Dynamics of a clamped-clamped microbeam resonator considering fabrication imperfections. Microsystem Technologies 21(11), 2425-2434 (2015)

5. Bellaredj, M., Bourbon, G., Walter, V., Le Moal, P., Berthillier, M.: Anodic bonding using soi wafer for fabrication of capacitive micromachined ultrasonic transducers. Journal of Micromechanics and Microengineering 24(2), 025009 (2014)

6. Braun, T., Barwich, V., Ghatkesar, M.K., Bredekamp, A.H., Gerber, C., Hegner, M., Lang, H.P.: Micromechanical mass sensors for biomolecular detection in a physiological environment. Physical Review E 72(3), 031907 (2005)

7. Brent, R.P.: Algorithms for minimization without derivatives. Courier Corporation (2013)

8. Celep, Z.: Free flexural vibration of initially imperfect thin plates with large elastic amplitudes. ZAMM-Journal of Applied Mathematics and Mechanics/Zeitschrift für Angewandte Mathematik und Mechanik 56(9), 423-428 (1976)

9. Celep, Z.: Shear and rotatory inertia effects on the large amplitude vibration of the initially imperfect plates. Journal of Applied Mechanics 47(3), 662-666 (1980)

10. Chu, W.H., Mehregany, M.: A study of residual stress distribution through the thickness of $\mathrm{p} / \mathrm{sup}+/$ silicon films (thermal oxidation effects). IEEE Transactions on Electron Devices 40(7), 1245-1250 (1993)

11. Comini, E., Faglia, G., Sberveglieri, G.: Solid state gas sensing, vol. 20. Springer Science \& Business Media (2008)

12. Duffy, S., Bozler, C., Rabe, S., Knecht, J., Travis, L., Wyatt, P., Keast, C., Gouker, M.: Mems microswitches for reconfigurable microwave circuitry. IEEE microwave and wireless components letters 11(3), 106-108 (2001)

13. Ganji, B.A., Majlis, B.Y.: Slotted capacitive microphone with sputtered aluminum diaphragm and photoresist sacrificial layer. Microsystem technologies 16(10), 1803-1809 (2010)

14. Hafiz, M.A.A., Kosuru, L., Younis, M.I.: Microelectromechanical reprogrammable logic device. Nature communications 7, 11137 (2016)

15. Hirsch, P., Roberts, S.: The brittle-ductile transition in silicon. Philosophical Magazine A 64(1), 55-80 (1991)

16. Jallouli, A., Kacem, N., Bourbon, G., Le Moal, P., Walter, V., Lardies, J.: Pull-in instability tuning in imperfect nonlinear circular microplates under electrostatic actuation. Physics Letters A 380(46), 3886-3890 (2016) 
17. Jallouli, A., Kacem, N., Lardies, J.: Investigations of the effects of geometric imperfections on the nonlinear static and dynamic behavior of capacitive micomachined ultrasonic transducers. Micromachines $\mathbf{9}(11), 575$ (2018)

18. Jallouli, A., Kacem, N., Najar, F., Bourbon, G., Lardies, J.: Modeling and experimental characterization of squeeze film effects in nonlinear capacitive circular microplates. Mechanical Systems and Signal Processing 127, 68 - 88 (2019)

19. Jia, X.L., Yang, J., Kitipornchai, S.: Pull-in instability of geometrically nonlinear micro-switches under electrostatic and casimir forces. Acta mechanica 218(1-2), 161-174 (2011)

20. Juillard, J., Bonnoit, A., Avignon, E., Hentz, S., Kacem, N., Colinet, E.: From mems to nems: Closed-loop actuation of resonant beams beyond the critical duffing amplitude. In: SENSORS, 2008 IEEE, pp. 510-513 (2008)

21. Kacem, N., Baguet, S., Hentz, S., Dufour, R.: Nonlinear phenomena in nanomechanical resonators: mechanical behaviors and physical limitations. Mechanics \& Industry 11(6), $521-529(2010)$

22. Kacem, N., Hentz, S., Baguet, S., Dufour, R.: Forced large amplitude periodic vibrations of non-linear mathieu resonators for microgyroscope applications. International Journal of Non-Linear Mechanics 46(10), 1347 - 1355w (2011)

23. Krylov, S., Ilic, B.R., Schreiber, D., Seretensky, S., Craighead, H.: The pull-in behavior of electrostatically actuated bistable microstructures. Journal of Micromechanics and Microengineering 18(5), 055026 (2008)

24. Mallik, S., Chowdhury, D., Chttopadhyay, M.: Development and performance analysis of a low-cost mems microphone-based hearing aid with three different audio amplifiers. Innovations in Systems and Software Engineering 15(1), 17-25 (2019)

25. Medina, L., Gilat, R., Krylov, S.: Bistable behavior of electrostatically actuated initially curved micro plate. Sensors and Actuators A: Physical 248, 193-198 (2016)

26. Medina, L., Gilat, R., Krylov, S.: Modeling strategies of electrostatically actuated initially curved bistable micro plates. International Journal of Solids and Structures (2017)

27. Medina, L., Gilat, R., Krylov, S.: Bistability criterion for electrostatically actuated initially curved micro plates. International Journal of Engineering Science 130, 75-92 (2018)

28. Naeli, K., Brand, O.: Dimensional considerations in achieving large quality factors for resonant silicon cantilevers in air. Journal of applied Physics 105(1), 014908 (2009)

29. Nayfeh, A.H., Balachandran, B.: Applied nonlinear dynamics: analytical, computational and experimental methods. John Wiley \& Sons (2008)

30. Ouakad, H.M.: An electrostatically actuated mems arch band-pass filter. Shock and Vibration 20(4), 809-819 (2013) 
31. Ouakad, H.M.: Simple and accurate analytical solution to the post-buckling response of an electrostatically actuated mems curled cantilever. Microsystem Technologies 22(9), $2251-2258(2016)$

32. Ouakad, H.M., Younis, M.I.: The dynamic behavior of mems arch resonators actuated electrically. International Journal of Non-Linear Mechanics 45(7), 704-713 (2010)

33. Rabenimanana, T., Walter, V., Kacem, N., Le Moal, P., Bourbon, G., Lardis, J.: Functionalization of electrostatic nonlinearities to overcome mode aliasing limitations in the sensitivity of mass microsensors based on energy localization. Applied Physics Letters $\mathbf{1 1 7}(3), 033502(2020)$

34. Rabenimanana, T., Walter, V., Kacem, N., Le Moal, P., Bourbon, G., Lardis, J.: Mass sensor using mode localization in two weakly coupled mems cantilevers with different lengths: Design and experimental model validation. Sensors and Actuators A: Physical 295, $643-652(2019)$

35. Rhoads, J.F., Shaw, S.W., Turner, K.L.: Nonlinear dynamics and its applications in micro-and nanoresonators. Journal of dynamic systems, measurement, and control 132(3) (2010)

36. Ruzziconi, L., Younis, M.I., Lenci, S.: An efficient reduced-order model to investigate the behavior of an imperfect microbeam under axial load and electric excitation. Journal of Computational and Nonlinear Dynamics 8(1) (2013)

37. Saghir, S., Bellaredj, M., Ramini, A., Younis, M.I.: Initially curved microplates under electrostatic actuation: theory and experiment. Journal of Micromechanics and Microengineering 26(9), 095004 (2016)

38. Saghir, S., Ilyas, S., Jaber, N., Younis, M.I.: An experimental and theoretical investigation of the mechanical behavior of multilayer initially curved microplates under electrostatic actuation. Journal of Vibration and Acoustics 139(4), 040901 (2017)

39. Saghir, S., Younis, M.I.: An investigation of the mechanical behavior of initially curved microplates under electrostatic actuation. Acta Mechanica 229(7), 2909-2922 (2018)

40. Sakhaee-Pour, A., Ahmadian, M., Vafai, A.: Applications of single-layered graphene sheets as mass sensors and atomistic dust detectors. Solid State Communications 145(4), 168-172 (2008)

41. Sazonova, V.A.: A tunable carbon nanotube resonator. Ph.D. thesis, Cornell University (2006)

42. Shmulevich, S., Lerman, M., Elata, D.: On the quality of quality-factor in gap-closing electrostatic resonators. Journal of Micromechanics and Microengineering 23(11), 115010 (2013) 
43. Thomas, O., Touzé, C., Chaigne, A.: Asymmetric non-linear forced vibrations of free-edge circular plates. part ii: experiments. Journal of Sound and Vibration 265(5), 1075-1101 (2003)

44. Touzé, C., Thomas, O., Chaigne, A.: Asymmetric non-linear forced vibrations of free-edge circular plates. part 1: Theory. Journal of Sound and Vibration 258(4), 649-676 (2002)

45. Walter, V., Bourbon, G., Le Moal, P., Kacem, N., Lardis, J.: Electrostatic actuation to counterbalance the manufacturing defects in a mems mass detection sensor using mode localization. Procedia Engineering 168, 1488 - 1491 (2016). Proceedings of the 30th anniversary Eurosensors Conference Eurosensors 2016, 4-7. Sepember 2016, Budapest, Hungary

46. Wang, Z., Ren, J.: Nonlinear coupled vibration of electrically actuated arch with flexible supports. Micromachines 10(11), 729 (2019)

47. Wang, Z., Ren, J.: Three-to-one internal resonance in mems arch resonators. Sensors $\mathbf{1 9}(8), 1888(2019)$

48. Williams, M.D., Griffin, B.A., Reagan, T.N., Underbrink, J.R., Sheplak, M.: An aln mems piezoelectric microphone for aeroacoustic applications. Journal of Microelectromechanical Systems 21(2), 270-283 (2012)

49. Younis, M.I.: MEMS linear and nonlinear statics and dynamics, vol. 20. Springer Science \& Business Media (2011)

50. Younis, M.I.: Analytical expressions for the electrostatically actuated curled beam problem. Microsystem Technologies 21(8), 1709-1717 (2015)

51. Younis, M.I., Ouakad, H.M., Alsaleem, F.M., Miles, R., Cui, W.: Nonlinear dynamics of mems arches under harmonic electrostatic actuation. Journal of Microelectromechanical Systems 19(3), 647-656 (2010)

52. Zhang, Y.: Large deflection of clamped circular plate and accuracy of its approximate analytical solutions. Science China Physics, Mechanics \& Astronomy 59(2), 624602 (2016) 